\title{
Perspectives of Certified Registered Nurse Anesthetists on Endoscopy Room Delays
}

\author{
Danny T. Y. Wu ${ }^{1,2^{*}}$ (D, Rohan Sachdeva1,3, Paul Murdock ${ }^{1,4}$, Scott Vennemeyer ${ }^{1,4}$, \\ Lisa Mattucci-Hunter ${ }^{5,6}$, Marcus Lehman ${ }^{5}$ \\ ${ }^{1}$ Department of Biomedical Informatics, College of Medicine, University of Cincinnati, Cincinnati, OH, USA \\ ${ }^{2}$ Department of Pediatrics, College of Medicine, University of Cincinnati, Cincinnati, OH, USA \\ ${ }^{3}$ College of Literature, Science, and the Arts, University of Michigan, Ann Arbor, MI, USA \\ ${ }^{4}$ Medical Sciences Baccalaureate Program, College of Medicine, University of Cincinnati, Cincinnati, OH, USA \\ ${ }^{5}$ Department of Anesthesiology, College of Medicine, University of Cincinnati, Cincinnati, OH, USA \\ ${ }^{6}$ CRNA Program, School of Nursing, University of Cincinnati, Cincinnati, OH, USA
}

Email: ^wutz@ucmail.uc.edu, tzuyu@umich.edu,rosach@umich.edu, murdockpaul96@gmail.com, vennemsm@mail.uc.edu, lisa.hunter@uc.edu,marcus.lehman@uc.edu

How to cite this paper: $\mathrm{Wu}$, D.T.Y., Sachdeva, R., Murdock, P., Vennemeyer, S., Mattucci-Hunter, L. and Lehman, M. (2021) Perspectives of Certified Registered Nurse Anesthetists on Endoscopy Room Delays. Open Journal of Nursing, 11, 947-959. https://doi.org/10.4236/ojn.2021.1111077

Received: September 13, 2021

Accepted: November 14, 2021

Published: November 17, 2021

Copyright (c) 2021 by author(s) and Scientific Research Publishing Inc. This work is licensed under the Creative Commons Attribution International License (CC BY 4.0).

http://creativecommons.org/licenses/by/4.0/

\begin{abstract}
As hospitals undergo reductions in budget and the demand for endoscopic procedures grows, the need for more efficient endoscopy units has increased. Certified Registered Nurse Anesthetists (CRNAs) play a vital role in Endoscopy Units and because of this, increasing CRNA work efficiency would increase the efficiency of the entire unit. This pilot study aims to explore CRNA workflow and identify workflow barriers present in endoscopy rooms through qualitative interview analysis. Ten semi-structured interviews were conducted at the University of Cincinnati Medical Center. The interview data were analyzed qualitatively to generate workflow diagrams and four themes of workflow barriers and representative quotes. These themes include: 1) physician unavailability, 2) patient delay and variability, 3) suboptimal teamwork and coordination, and 4) issues with supporting tools. The workflow barriers reflect and extend the current literature, and can inform future quality improvement initiatives.
\end{abstract}

\section{Keywords \\ CRNA, Workflow, Efficiency, Endoscopy, Qualitative}

\section{Introduction}

Certified Registered Nurse Anesthetists (CRNAs) are responsible for providing anesthesia to patients in many units across a hospital. Generally, CRNAs are required to receive training from an accredited nurse anesthesia program, and are 
an integral part of the anesthesia landscape within the United States (US) as they are often attending to a sedated patient as a sole provider or under the supervision of an anesthesiologist, depending on the practice model of the facility [1]. With well-designed training programs, CRNAs can be great resources to improve care access and delivery as well as patient experience and safety [2] [3] [4]. Similar to other surgical specialties and settings, CRNAs are present in endoscopy units to provide anesthesia for endoscopic procedures. As newer and more intricate procedures are developed, the demand for these endoscopic procedures is increasing. Studies suggest that the demand for colonoscopies in the US would require an increase of 32,700 gastroenterologists every ten years nationwide [5].

While the demand for endoscopy-related procedures is increasing, delays and a lack of efficiency are common within endoscopy units due to the large amount of moving actors present within the unit, especially the flow of patients in and out of the hospital [6]. As hospitals attempt to optimize budgets along with the increasing demand for endoscopic procedures, it is critical to ensure efficiency within an endoscopy unit through structure, processes, and outcomes to conserve and improve the efficiency and quality of care [7]. Moreover, resources such as personnel and expensive equipment are often under-utilized in endoscopy units, and mismanagement of these resources in larger academic health systems is more prevalent [7] [8] [9]. Improving efficiency when administering anesthesia seems to be correlated to higher patient satisfaction as well [10]. Exploring the causes of delay within an endoscopy unit would be a critical first step to improve the quality of anesthesia care.

Studies have identified major efficiency barriers, including patients punctuality and delays caused by both patients and physicians, which may be resolved by dynamic scheduling [11] [12] [13]. While many papers examined workflow issues in endoscopy units, little research has been done regarding the delay experienced by a Certified Registered Nurse Anesthetist (CRNA). The perspective of CRNAs on workflow efficiency is critical, because CRNAs are likely the most affected group when considering delays in endoscopy processes. This is because CRNAs are required to stay with the patient while they are sedated and help with the room turnover by ensuring that all equipment has been properly cleaned and set up prior to the next procedure.

To understand workflow processes and examine workflow issues, a time-motion study is frequently conducted. However, these studies are often time and resource consuming; these issues were further exacerbated by the COVID-19 pandemic as in-person shadowing, and direct observations are much more difficult or even prohibited to conduct. Qualitative studies and content analysis have been a trustworthy method in nursing research [14] and can be a viable alternative to identify thematic barriers of workflow issues. The results of the qualitative analysis can inform future time-motion studies and focus on capturing and qualifying certain workflow issues.

In this study, the research team aimed to gain a better understanding of the current workflow, subsequent efficiency barriers, and delays perceived by CRNAs 
in an endoscopy unit through semi-structured interviews. Our research questions were twofold. First, what were the workflow and CRNA-centered processes of an endoscopy unit? Second, what were the common workflow barriers from CRNAs' perspectives and what were the potential solutions? The findings of the present study can help us develop future quantitative and mix-method studies and guide quality improvement initiatives.

\section{Methods}

\subsection{Clinical Setting}

This study was conducted at the University of Cincinnati Medical Center (UCMC), an academic medical center within the UC Health System. The study was approved in April 2018. The digestive diseases group spans across the Greater Cincinnati area, with two locations in Clifton and West Chester, Cincinnati. Members of Digestive Diseases collaborate with surgical specialists, pediatric gastroenterology specialists at Cincinnati Children's Hospital Medical Center, and other clinicians to provide a high quality of multidisciplinary care in the areas of pancreas diseases, liver disorders, inflammatory bowel disease and interventional endoscopy. The endoscopy unit of the digestive diseases group at UCMC was chosen as the primary clinical setting for the present study. Currently, 15 - 20 CRNA's work in the endoscopy unit, half of which have a regular schedule in the unit.

\subsection{Study Design}

The study began with recruiting a group of CRNAs through a purposive sampling technique, based on collaborator recommendation. Specifically, the participants were chosen based on the physician collaborator's professional network and recommendation, considering the CRNAs' years of experience and availability for an interview. Each interview was conducted in-person in a semi-structured format and lasted for about 30 minutes. These interviews were conducted between April and July of 2018. The interviewers asked questions regarding the daily workflow and perceived bottleneck issues that can affect workflow efficiency. The interview questions were organized in the following four areas: 1) what are your job title and responsibilities? 2) what does a typical workday look like? 3) what factors are affecting your workflow efficiency? and 4) how would you address the workflow barriers and issues? The interviews were recorded and transcribed verbatim for further analysis by the research team. The interview transcripts were reviewed by a team member to ensure the quality and accuracy of the information. The transcripts were de-identified, and the participants were referred to using a unique serial identifier. This study was reviewed and approved by the UC Institutional Review Board (IRB\# 2018-1120).

\subsection{Participant Recruitment}

Ten CRNAs were recruited before the COVID-19 pandemic through a conveni- 
ence sampling methodology based on anesthesiologist and manager referral. This sample size was selected as it is representative of the whole unit and reached data saturation. Participants were numbered from "P01" to "P10".

\subsection{Data Analysis}

The transcribed interview data were analyzed in two separate and parallel steps. First, the transcripts were coded using the workflow elements model [15] and a swim line diagram was constructed for each participant. In each workflow diagram, specific lanes were used to describe the processes and outcomes of each clinical role based on the interview data. Rectangular ovals were used to represent actions; diamonds were used for decisions; circles were used to indicate the starting point and end point of the workflow. In the second step, the transcripts were analyzed in a bottom-up manner to generate categories and themes of workflow barriers and issues. Specifically, significant quotes indicating bottleneck issues were transcribed to sticky notes, and an affinity diagram was created through two sessions to identify and group the perceived workflow issues (Table 2).

\section{Results}

The participants had an equal split in gender, and their time at current position and daily patient volume varied. The variation in daily patients was mostly likely due to the specific shift and schedule of each CRNA. The participant demographics are summarized in Table 1.

Figure 1 shows the consolidated workflow of the participating CRNAs. CRNAs generally started their day around 7a.m. by preparing the room and conducting pre-operation with the anesthesiologist while also conducting drug preparation and other surgical preparations. At the same time, the registered nurses (RN team hereafter) obtain consent from the patient. If the RN team fails to obtain consent, the endoscopist will be required to obtain it. After consent is obtained, the sedative is administered by the CRNA. The sedation the CRNA provides differs depending on the procedure. For example, a colonoscopy or an esophagogastroduodenoscopy (EGD) requires a monitored sedation while other procedures may require different anesthetic methods. While the CRNA sedates the patient, the RN team documents the procedure, and the anesthesiologist is responsible for overseeing the delivery of the sedative. After the sedative has been administered, the endoscopist begins the procedure. When the procedure is done, the

Table 1. Summary of participant characteristics.

\begin{tabular}{cc}
\hline Characteristic & No. (\%) \\
\hline Male & $5(50 \%)$ \\
Female & $5(50 \%)$ \\
Mean Time at Current Position & 5.4 years \\
Mean Daily Patient Volume & 6.44 patients
\end{tabular}




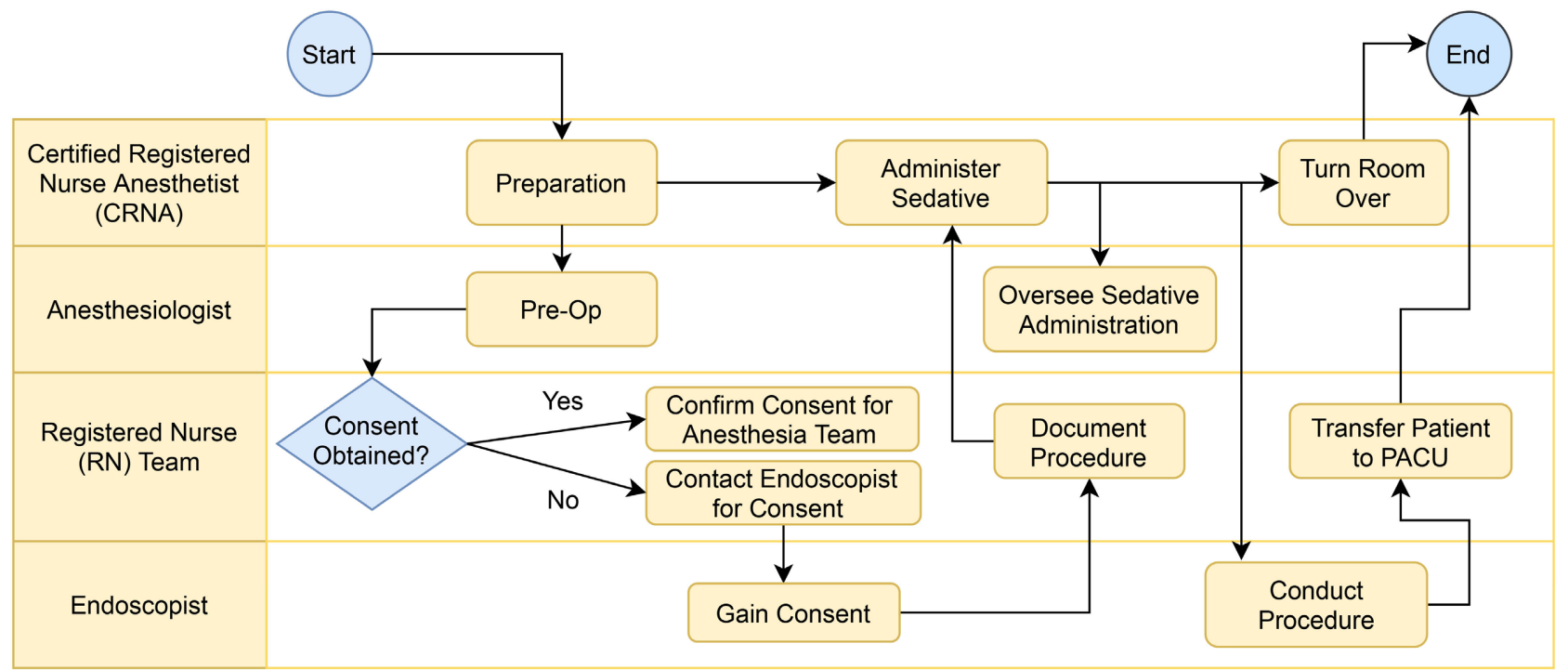

Figure 1. Consolidated workflow diagram of the participating CRNAs. Legend: Rectangular Oval = action; Diamond = decision; circle $=$ start/end of workflow.

$\mathrm{RN}$ team is responsible for transferring the patient to a post-anesthesia care unit (PACU) to recover. At the same time, the CRNA is responsible for "turning the room over," which includes cleaning of endoscopic instruments, drug preparation, and other tasks to get the room ready for the next patient.

\section{Workflow Barriers}

The affinity diagram indicated four main themes of workflow barriers, including: 1) physician unavailability, 2) patient delay and variability, 3) suboptimal teamwork and coordination, and 4) issues with supporting tools. These barriers and their associated categories are outlined in Table 2, which also states a definition for each category and documents the number of participants that mentioned this category as an issue.

\section{Theme 1: Physician Unavailability}

Examining the interview data revealed that physician unavailability (or delay) was often mentioned as the largest source of workflow delay for the CRNAs. Delving deeper into these issues revealed three major reasons for such delay: inflexible scheduling, general lateness on the part of the physician, and teaching load. Inflexible and inefficient scheduling can often lead to physician delay as physicians can be scheduled with little to no downtime between cases or can be overscheduled, which leads to delays as the whole schedule can be thrown off if one case is delayed. Scheduling back-to-back cases for the same doctor in different rooms can also lead to delay due to travel time. The following two quotes evidence this claim:

"Seems like they try to build the schedule so there's the least amount of time in between cases... One more frustrating delay is when they have one doctor doing multiple cases, they might have to physician one case before going onto the next one in a different room and it happens and is aggravating." (P07). 
Table 2. Themes and categories of the workflow barriers.

\begin{tabular}{ccc}
\hline Theme & Number of \\
& $\begin{array}{c}\text { Participants } \\
\text { mentioned }\end{array}$ & Definition/Phenomenon \\
\hline
\end{tabular}

\begin{tabular}{|c|c|c|c|}
\hline \multirow{3}{*}{$\begin{array}{l}\text { Physician } \\
\text { Unavailability }\end{array}$} & Inflexible scheduling & 8 & $\begin{array}{l}\text { Physicians can often come in late from another procedure due to } \\
\text { inflexible scheduling. This occurs when a physician is doing multiple } \\
\text { cases in different rooms or cases are scheduled too close together. }\end{array}$ \\
\hline & Personal reasons & 8 & $\begin{array}{l}\text { The procedure cannot begin until the doctor is present at the } \\
\text { procedure, ensuring that the entire schedule of the day is delayed if } \\
\text { they are not present. This specific group accounts for general } \\
\text { lateness on the part of the physician due to personal reasons, } \\
\text { habitually arriving late in the morning, etc. }\end{array}$ \\
\hline & Teaching load & 4 & $\begin{array}{l}\text { Because of UCMC as a teaching hospital, residents are often doing } \\
\text { procedures. Having residents help with procedures may significantly } \\
\text { delay them. }\end{array}$ \\
\hline \multirow{2}{*}{$\begin{array}{l}\text { Patient Delay } \\
\text { and Variability }\end{array}$} & Patient additions & 7 & $\begin{array}{l}\text { Patients are often added to the schedule unexpectedly, which can } \\
\text { delay all future cases and throw off the entire schedule. }\end{array}$ \\
\hline & $\begin{array}{l}\text { Patient arrives } \\
\text { late or no show }\end{array}$ & 7 & $\begin{array}{l}\text { Patients can often arrive late for a procedure or not show up at all, } \\
\text { which causes uncontrollable delay. }\end{array}$ \\
\hline \multirow{3}{*}{$\begin{array}{l}\text { Suboptimal } \\
\text { Teamwork and } \\
\text { Coordination }\end{array}$} & Communication issues & 8 & $\begin{array}{l}\text { Communication issues wit in a team and/or a lack of direct } \\
\text { communication between team members in the endoscopy room } \\
\text { can lead to delays. }\end{array}$ \\
\hline & Inefficient room shifts & 5 & $\begin{array}{l}\text { Schedules are often crammed with inefficient room shifts, which can } \\
\text { contribute to delay as the delay caused by these room shifts can } \\
\text { throw off the schedule. }\end{array}$ \\
\hline & Room turnover & 5 & $\begin{array}{l}\text { Delays in turning over rooms can resultantly throw off the entire } \\
\text { schedule. This may be due to low staffing. }\end{array}$ \\
\hline \multirow{3}{*}{$\begin{array}{l}\text { Issues with } \\
\text { Supporting Tools }\end{array}$} & $\begin{array}{l}\text { Issues with } \\
\text { physical equipment }\end{array}$ & 6 & $\begin{array}{l}\text { Delay can be caused when equipment malfunctions, is not properly } \\
\text { cleaned, or has other issues. }\end{array}$ \\
\hline & $\begin{array}{l}\text { Supply issues/improper } \\
\text { stocking }\end{array}$ & 4 & $\begin{array}{l}\text { Often supplies are understocked or unnecessarily overstocked. The } \\
\text { distance between Endo and the OR Pharmacy causes issues as it is } \\
\text { difficult and time-consuming to check the cart for what supplies are } \\
\text { already stocked and get the supplies necessary. }\end{array}$ \\
\hline & EMR system malfunction & 2 & $\begin{array}{l}\text { The EMR system is used for digital note-taking but has been } \\
\text { described as "non-intuitive" and "painful" when it malfunctions. } \\
\text { When EMR goes down, the notes must be manually entered, which } \\
\text { is a major hindrance. }\end{array}$ \\
\hline
\end{tabular}

"Also, sometimes weird things with scheduling, like if they have two here and two there. Like if they book a patient before they know they'll be done and if the surgeon is running late, you'll be even more delayed. Or throwing non-anesthesia patients in with anesthesia patients." (P08)

It was also found that physicians could be delayed due to a myriad of other reasons, including personal reasons or general lateness, which could add to de- 
lay.

“...surgeon delays, one particular surgeon who does not show up at 7:30 but is there by 7:45 normally." (P04)

Physician delay may be contributed to by the teaching load present, or by the inexperience of residents doing procedures. As a result of UC's status as a teaching hospital, residents are often doing procedures, which can significantly delay the entire schedule. One participant described the physician unavailability that teaching load can cause, explaining that:

"EGDs usually take less than 20 minutes, and colons are only supposed to take about 20 minutes, but that can last up to an hour and a half here. Because this is a teaching hospital, so you have the residents doing a lot of them". (P01)

\section{Theme 2: Patient Delay and Variability}

Our participants cited patient delay as another significant source of delay. This barrier seems to be quite sporadic as it is mostly based upon patient variability. This theme was categorized as delay caused by patient add-ons, or delay that is caused when patients arrive late or never show up. Patients are often added to the schedule unexpectedly, which can delay all future cases and throw off the entire schedule. The reasons for adding patients to the schedule vary from transfers due to procedure limitations at another hospital to inpatient add-ons who are having issues that need immediate attention:

"For example, the doctor will have an EGD, EUS scheduled at 12 but we'll have a transfer from the VA, because they don't do ERCPs over there, I don't think. So, they'll be an add-on from the floor or the VA, and we'll have 2 or even 3 add-ons during the day. So, you never know what's going on. A lot of times, it looks like your day may end at 11 am in endo, but it can go as late as 5:00 (pm) because of add-ons." (P01)

Another participant also discussed inefficient additions to the schedule, explaining that big cases can be fit into the schedule at inappropriate times, which causes further delay by throwing off the entire schedule:

"I think there are scheduling snafu's so I just think inappropriate scheduling also, we have some guys that will just put on a myriad of stuff at the end of the day. Like big cases like ERCP's."(P10)

The other major type of patient delay occurs when patients arrive late or fail to show up for the procedure at all. This delay can similarly throw off the entire schedule and push cases back. One participant explained that these delays are out of the participants' control, stating that there were:

"always patient delays. If they' re running behind or status changes, such as if they just ate, but those things are generally out of our control' (P04).

As the above quote explains, patient variability plays a part as patients can cause delay by simply not following the directions they are given, such as refraining from eating, which can cause further delay. Another participant further evidenced this claim, explaining that downtime is caused when patients fail to show up for their appointments: 
"I feel like there's a lot of down time when patients aren't showing up. I had one day, I think it was last week or two weeks ago, where the first patient didn't show up and so they were trying to call for another one and there weren't many inpatients."(P05)

Theme 3: Suboptimal Teamwork and Coordination

Our participants discussed issues related to teamwork and coordination between the CRNA's and the other units in the Endoscopy area, including a lack of communication between actors in an endoscopy unit, inefficient room shifts, and room turnover delay. Communication issues between the participant CRNA's and the nurses and doctors were listed as a major barrier to workflow efficiency that tended to cause delays regarding patient transportation or room turnover. These communication issues generally occur when a participant is unable to contact a provider, or has to go find a doctor and/or nurse prior to a procedure. Participant P08 explained that this is an issue, also stating that other areas of the hospital have communication systems but in the endoscopy unit, CRNAs often have to physically chase people down:

"In the main $O R$ we have a different system to let everyone know we' re ready. The way Epic is it has it change colors which notifies us they're ready to bring the patient back. We also have to click a surgeon logo and if $P m$ way far away I can see if they' re ready and if the surgeon is here, whereas in endo you' re walking around trying to find people. I think it would be helpful and feasible, might be more efficient than walking around trying to find people." (P08).

Another participant also elaborated upon communication issues in the endoscopy unit, explaining that downtime could be prevented if CRNAs had more advanced notice for when they have a patient:

"You need to give someone more heads up before sticking someone in their room. Could say hey are you doing anything, are you free so we can stick someone in your room instead of saying " Hey we re doing an EGD in your room right now." (P03).

Another category of delay includes inefficient room shifts that can cause confusion and delays by interrupting the workflow in each room. This includes shifting the CRNAs themselves as well as shifting other actors within the endoscopy area such as nurses. One participant did not understand some nursing shifts, and explained as follows. This participant further states that staying in "just one room is easier because you get yourself into a flow".

"Nursing staff that they'll shift. Im sure there's a reason but I don't understand it but they'll shift someone from a room to being in PACU [Post-Anesthesia Care Unit] or from room to room or cleaning a scope room to being in a room and I don't always understand it" as he feels it can "lead to difficulties" (P09).

Still another category of delay is room turnover time. The participant's explanation behind the reasons for room turnover delay include a lack of personnel necessary to efficiently change the room and slow working physicians. In fact, the CRNAs explained that room turnover is left solely in the hands of the CRNA themselves and that no one is present in the endoscopy unit to help turnover 
rooms, which could help explain this source of delay:

"Specifically in the room where we do bronchoscopies, the turnover rate is very long, Id say 45 minutes. Mostly because there's a lot of equipment that needs to be cleaned. If they had an extra person to help do that it might help speed it up. If you have four procedures in that room that's a couple hours of downtime" (P07).

"Then they'll do the case, and when they' re done, we'll turn off the anesthetic, take the patients off the monitor, clean the equipment, since there isn't a tech for us over there, and then when that's done, Ill take the patient over to the recovery room and get them hooked back up, talk to the nurse there. And hopefully in that time I have been able to set up for my next patient, so that should all be ready to go and get back there" (P01).

\section{Theme 4: Issues with Supporting Tools}

A fourth major issue arises when equipment, either physical or digital, malfunctions or is not properly taken care of. One frequently mentioned workflow barrier occurred when "scopes" were not cleaned prior to the procedure. Many CRNAs mentioned a source of downtime being a lack of available scopes, with one stating that,

"Sometimes, we get delayed because they don't have enough scopes or the scopes aren't clean. I don't know how many they have, but it's not something that I deal with. Sometimes, I feel that we are waiting on a scope to be cleaned, and that can slow us down." (P02)

Others also cited general equipment malfunction as a major issue; P02 mentioned that the fluoroscopy equipment often malfunctions:

"Another issue that happens down there sometimes is equipment issues. There's a room down there with all new fluoroscopy equipment, that I feel that something malfunctions, not every day, but often." (P02)

Furthermore, another equipment issue centers around improper stocking of supplies. Supplies such as $100 \mathrm{ml}$ propofol and vasopressors often end up either overstocked or understocked because of the large distance between the Endoscopy unit and the OR pharmacy. Generally, an anesthesia tech is responsible for restocking the cart, but when done improperly a workflow barrier is created for the CRNA's.

"Probably my biggest hurdle, from an anesthesia standpoint, is when the machines aren't stocked appropriately. I believe it is the night shift anesthesia tech that is supposed to restock the cart. Usually, I get there little early in the morning and I just double check everything. But really, it should be there. It doesn't cause wasted time, because I get in early and get everything before the case even starts. I would say it really affects the flow. It's just frustrating." (P06)

Issues with the EMR documentation system were mentioned as a barrier for workflow when the system malfunctioned. The EMR system is used for digital note-taking but has been described as "non-intuitive" and "painful" when it malfunctions. When the EMR system goes down, the notes must be manually 
entered, which is time-consuming and a major hindrance. One participant shared this sentiment, explaining that:

"The only thing that is even a hiccup...I mean we've had some issues with the computers... the EPIC talking to the system that gathers the vitals. It's fixed now, but occasionally it ll go out or go wonky. If that happens, it hasn't happened that much but when it does happen that is a major hindrance to work from, if there's any hiccups with that." (P09).

\section{Discussion}

This study identified and outlined potential bottleneck issues that affect the efficiency of CRNAs in an endoscopy unit within a large academic institution. Qualitative analysis revealed four major thematic barriers: physician unavailability, patient delay and variability, suboptimal teamwork and coordination, and issues with supporting tools. These barriers were further divided into categories, with some of the most prevalent sub-groups being inflexible scheduling, patient additions, communication issues, or delay due to lack of physician punctuality for operations.

Our findings corroborate and extend current literature on workflow issues present in similar units. First, it is not surprising that CRNAs have communication issues in their team-based clinical work. A work habit survey showed that $60 \%$ of negative comments were associated with specific themes of work habit items, and that the most frequent themes were communication-related [16]. CRNAs likely experienced many interruptions and distractions during their work (68 times per hour), which may come from other personnel, conversation, and noise [17]. While some conversations may be needed, unnecessary interruptions and distractions should be identified and eliminated to improve communication effectiveness and workflow efficiency. Second, patient delays and no shows are out of CRNAs' hands but can be addressed partially by target messaging, phone calls, dedicated medical notes, and flexible scheduling [18] [19]. Physician unavailability, one the other hand, seems to be a major factor contributing to workflow inefficiency and procedural delays in endoscopy units [6] [11]. Initiatives and policies can be implemented to improve physicians' availability and adherence to their schedules, and changing staffing ratio and increasing shared block times are other viable solutions [20] [21] [22]. Finally, issues with supporting tools including equipment, stocking, and EMR malfunction are less documented in the literature as a major factor in the workflow efficiency of CRNAs. It seems that CRNAs have to physically chase down colleagues and equipment. A real-time locating system may reduce the tracking effort and help collect and quantify workflow delays and further streamline the processes [23]. Moreover, EMR usability and unintended consequences of health information technologies have been a major research topic in medical informatics [24] [25] and should be researched further with a focus on CRNAs and endoscopy units. Other supporting tools, such as routine checklists, may be utilized, but their clinical utility and 
impact on workflow should be carefully planned and evaluated [26].

This study has several limitations. First, since this study only interviewed 10 CRNAs at one institution, the generalizability of the study is limited, which is inherent in a qualitative study. However, as discussed above, our findings reflect the literature and bridge the knowledge gaps by identifying additional themes of workflow barriers. It is important to note that the small sample of the participants is still representative of the population of nurse anesthetists in our study, as only about 15 to 20 CRNAs work in the endoscopy unit of our site. Second, no quantitative data was gathered to quantify the delay. We used the present study as a critical first step to understand CRNA's workflow and delays and will collect quantitative data in the next phase of the study. Third, the study was conducted before the COVID-19 pandemic. The workflow barriers and care delivery challenges of CRNAs during and in the late stage of the pandemic may be different from our current findings.

In future work, we will address these limitations by conducting quantitative data collection and analysis and gathering data from other institutions. Timestamps from time-motion studies, electronic health records, and even real-time locating systems will be utilized to quantify the delays present for CRNAs. We also plan to develop a novel workflow monitoring system to provide a comprehensive picture on workflow barriers and create a timely feedback system for leadership to review and take actions.

\section{Conclusion}

This study examined the workflow barriers perceived by CRNAs in an Endoscopy unit and generated workflow diagrams along with four themes of barriers. This study confirmed that workflow efficiency is an issue present for CRNAs in our endoscopy unit, and the findings can be valuable lessons to other institutions. We will continue studying this issue and work with the clinical team and the leadership to develop quality improvement initiatives to reduce workflow delays, improve care quality, and further improve staff and patient satisfaction in our endoscopy units and anesthesiology services.

\section{Acknowledgements}

We thank Ms. Samantha Jarzynka for her effort on interview data analysis.

\section{Disclosures}

The authors have declared no financial relationships with any commercial entity related to the content of this article.

\section{Conflicts of Interest}

The authors declare no conflicts of interest regarding the publication of this paper. 


\section{References}

[1] Matsusaki, T. and Sakai, T. (2011) The Role of Certified Registered Nurse Anesthetists in the United States. Journal of Anesthesia, 25, 734-740. https://doi.org/10.1007/s00540-011-1193-5

[2] Bortman, J., Mahmood, F., Mitchell, J., et al. (2019) Ultrasound-Guided Intravenous Line Placement Course for Certified Registered Nurse Anesthetists: A Necessary Next Step. AANA Journal, 87, 269-275.

[3] Ostrowski, A.M., Morrison, S. and O’Donnell, J. (2019) Development of a Training Program in Peripherally Inserted Central Catheter Placement for Certified Registered Nurse Anesthetists Using an N-of-1 Method. AANA Journal, 87, 11-18.

[4] Jordan, L. (2011) Studies Support Removing CRNA Supervision Rule to Maximize Anesthesia Workforce and Ensure Patient Access to Care. AANA Journal, 79, 101-104.

[5] Vijan, S., Inadomi, J., Hayward, R.A., Hofer, T.P. and Fendrick, A.M. (2004) Projections of Demand and Capacity for Colonoscopy Related to Increasing Rates of Colorectal Cancer Screening in the United States. Alimentary Pharmacology \& Therapeutics, 20, 507-515. https://doi.org/10.1111/j.1365-2036.2004.01960.x

[6] Yang, D., Summerlee, R., Suarez, A.L., Perbtani, Y., Blair Williamson, J., Shrode, C.W., et al. (2016) Evaluation of Interventional Endoscopy Unit Efficiency Metrics at a Tertiary Academic Medical Center. Endoscopy International Open, 4, E143-E148. https://doi.org/10.1055/s-0041-108082

[7] Gellad, Z.F., Thompson, C.P. and Taheri, J. (2013) Endoscopy Unit Efficiency: Quality Redefined. Clinical Gastroenterology and Hepatology, 11, 1046-1049.e1. https://doi.org/10.1016/j.cgh.2013.06.005

[8] Petersen, B.T. (2006) Promoting Efficiency in Gastrointestinal Endoscopy. Gastrointestinal Endoscopy Clinics of North America, 16, 671-685.

https://doi.org/10.1016/j.giec.2006.08.011

[9] Kowalski, T., Edmundowicz, S. and Vacante, N. (2004) Endoscopy Unit Form and Function. Gastrointestinal Endoscopy Clinics of North America, 14, 657-666, viii. https://doi.org/10.1016/j.giec.2004.04.006

[10] Navidi, B. and Kiai, K. (2019) Efficiency and Scheduling in the Nonoperating Room Anesthesia Suite: Implications from Patient Satisfaction to Increased Revenue Operating Room: A Common (Dollars and Sense) Approach. Current Opinion in Anaesthesiology, 32, 498-503. https://doi.org/10.1097/ACO.0000000000000744

[11] Yong, E., Zenkova, O., Saibil, F., Cohen, L.B., Rhodes, K. and Rabeneck, L. (2006) Efficiency of an Endoscopy Suite in a Teaching Hospital: Delays, Prolonged Procedures, and Hospital Waiting Times. Gastrointestinal Endoscopy, 64, 760-764. https://doi.org/10.1016/j.gie.2006.02.047

[12] Creps, J. and Lotfi, V. (2017) A Dynamic Approach for Outpatient Scheduling. Journal of Medical Economics, 20, 786-798. https://doi.org/10.1080/13696998.2017.1318755

[13] Ramos, C. and Gress, F. (2016) Tackling No-Shows and Improving Endoscopy Suite Efficiency. Gastrointestinal Endoscopy, 84, 1018-1020.

https://doi.org/10.1016/j.gie.2016.07.034

[14] Graneheim, U.H. and Lundman, B. (2004) Qualitative Content Analysis in Nursing Research: Concepts, Procedures and Measures to Achieve Trustworthiness. Nurse Education Today, 24, 105-112. https://doi.org/10.1016/j.nedt.2003.10.001

[15] Unertl, K.M., Novak, L.L., Johnson, K.B. and Lorenzi, N.M. (2010) Traversing the Many Paths of Workflow Research: Developing a Conceptual Framework of Workflow 
Terminology through a Systematic Literature Review. Journal of the American Medical Informatics Association, 17, 265-273.

https://doi.org/10.1136/jamia.2010.004333

[16] Dexter, F., Ledolter, J. and Hindman, B.J. (2017) Validity of Using a Work Habits Scale for the Daily Evaluation of Nurse Anesthetists' Clinical Performance While Controlling for the Leniencies of the Rating Anesthesiologists. Journal of Clinical Anesthesia, 42, 63-68. https://doi.org/10.1016/j.jclinane.2017.08.002

[17] Pape, T.M. and Dingman, S.K. (2011) Interruptions and Distractions during Anesthesia Induction: A Pilot Study. Plastic Surgical Nursing, 31, 49-56. https://doi.org/10.1097/PSN.0b013e31821b5b84

[18] Rosenbaum, J.I., Mieloszyk, R.J., Hall, C.S., Hippe, D.S., Gunn, M.L. and Bhargava, P. (2018) Understanding Why Patients No-Show: Observations of 2.9 Million Outpatient Imaging Visits Over 16 Years. Journal of the American College of Radiology, 15, 944-950. https://doi.org/10.1016/j.jacr.2018.03.053

[19] Kogan-Liberman, D., Rivas, Y., Thompson, J. and Tomer, G. (2015) Improving Nonattendance at Outpatient Pediatric Endoscopy Unit of a Tertiary Center. Journal of Pediatric Gastroenterology and Nutrition, 61, 234-237. https://doi.org/10.1097/MPG.0000000000000791

[20] Chen, Y., Gabriel, R.A., Kodali, B.S. and Urman, R.D. (2016) Effect of Anesthesia Staffing Ratio on First-Case Surgical Start Time. Journal of Medical Systems, 40, Article No. 115. https://doi.org/10.1007/s10916-016-0471-Z

[21] Tsai, M.H., Cipri, L.A., O’Donnell, S.E., Matthew Fisher, J. and Andritsos, D.A. (2017) Scheduling Non-Operating Room Anesthesia Cases in Endoscopy: Using the Sandbox Analogy. Journal of Clinical Anesthesia, 40, 1-6. https://doi.org/10.1016/j.jclinane.2017.03.010

[22] Epstein, R.H. and Dexter, F. (2017) Workload, Efficiency, and Productivity Following Open Access Scheduling in a Gastrointestinal Endoscopy Suite. Journal of Clinical Anesthesia, 40, 88-90. https://doi.org/10.1016/j.jclinane.2017.03.035

[23] Overmann, K.M., Wu, D.T.Y., Xu, C.T., Bindhu, S.S. and Barrick, L. (2021) Real-Time Locating Systems to Improve Healthcare Delivery: A Systematic Review. Journal of the American Medical Informatics Association, 28, 1308-1317. https://doi.org/10.1093/jamia/ocab026

[24] Coiera, E., Ash, J. and Berg, M. (2016) The Unintended Consequences of Health Information Technology Revisited. Yearbook of Medical Informatics, 25, 163-169. https://doi.org/10.15265/IY-2016-014

[25] Colicchio, T.K., Cimino, J.J. and Del Fiol, G. (2019) Unintended Consequences of Nationwide Electronic Health Record Adoption: Challenges and Opportunities in the Post-Meaningful Use Era. Journal of Medical Internet Research, 21, Article ID: e13313. https://doi.org/10.2196/13313

[26] Krombach, J.W., Edwards, W.A., Marks, J.D. and Radke, O.C. (2015) Checklists and Other Cognitive Aids for Emergency and Routine Anesthesia Care-A Survey on the Perception of Anesthesia Providers from a Large Academic US Institution. Anesthesia and Pain Medicine, 5, Article ID: e26300.

https://doi.org/10.5812/aamp.26300v2 